\title{
Rational Calogero-Moser Model: Explicit Form and $r$-Matrix of the Second Poisson Structure
}

\author{
Jean $A V A N^{\dagger}$ and Eric RAGOUCY $\ddagger$ \\ † Laboratoire de Physique Théorique et Modélisation, \\ Université de Cergy-Pontoise (CNRS UMR 8089), Saint-Martin 2, \\ 2, av. Adolphe Chauvin, F-95302 Cergy-Pontoise Cedex, France \\ E-mail: avan@u-cergy.fr \\ $\ddagger$ LAPTH Annecy le Vieux, CNRS and Université de Savoie, \\ 9 chemin de Bellevue, BP 110, F-74941 Annecy-le-Vieux Cedex, France \\ E-mail: ragoucy@lapp.in2p3.fr
}

Received July 24, 2012, in final form October 17, 2012; Published online October 26, 2012 http://dx.doi.org/10.3842/SIGMA.2012.079

\begin{abstract}
We compute the full expression of the second Poisson bracket structure for $N=2$ and $N=3$ site rational classical Calogero-Moser model. We propose an $r$-matrix formulation for $N=2$. It is identified with the classical limit of the second dynamical boundary algebra previously built by the authors.
\end{abstract}

Key words: classical integrable systems; hierarchy of Poisson structures; dynamical reflection equation

2010 Mathematics Subject Classification: 81R12; 16T15; 16T25

\section{Introduction}

\subsection{Dynamical $r$-matrices}

The Calogero-Moser model [13, 14, 23] provided [7] a textbook example of a classical dynamical $r$-matrix structure for a Lax representation [19] of a classical Liouville integrable system. Remember that Liouville integrability for a $N$-dimensional Hamiltonian system is characterized (see [8] and references therein) by the existence of $N$ independent Poisson-commuting quantities $H^{(i)}, i=1, \ldots, N$ including the original Hamiltonian. The system admits a Lax representation when the equations of motion can be represented as a spectrum-preserving evolution of a $n \times n$ matrix $L$ encapsulating the $2 N$ dynamical variables $\left\{p_{i}, q_{i}\right\}, i=1, \ldots, N$,

$$
\frac{d L}{d t}=[L, M]
$$

The quantities $\operatorname{Tr} L^{k}, k=1, \ldots, n$ may then provide the Liouville Hamiltonians if they Poisson-commute and they are in sufficient number (e.g. if $n \geq N$ or there exists a spectralparameter dependance). They build in this case a so-called Hamiltonian hierarchy of mutually compatible equations of motion. This Poisson-commuting property is equivalent [9] to rewriting the Poisson brackets of the Lax matrix coefficients in a specific algebraic form involving a matrix $r$, living in the tensor product $M_{n}(\mathbb{C}) \otimes M_{n}(\mathbb{C}), M_{n}(\mathbb{C})$ being self-explanatorily the algebra of complex $n \times n$ matrices

$$
\left\{L_{1}, L_{2}\right\}=\left[r_{12}, L_{1}\right]-\left[r_{21}, L_{2}\right]
$$


Expression (2) is the so-called linear form of $r$-matrix structure. Associativity of the Poisson bracket form (2) is guaranteed by the consistency equation

$$
\left[r_{12}, r_{13}\right]+\left[r_{12}, r_{23}\right]+\left[r_{32}, r_{13}\right]+\left\{r_{12}, L_{3}\right\}-\left\{r_{13}, L_{2}\right\}=0
$$

generically known as "dynamical Yang-Baxter equation" (dYB), see e.g. [22]. If $r$ does not depend on the dynamical variables (3) becomes a purely algebraic "classical Yang-Baxter equation". If not the issue arises of an available algebraic reexpression of the dynamical contribution $\left\{r_{12}, L_{3}\right\}-\left\{r_{13}, L_{2}\right\}$. At least two such forms are available in the literature. The first one occurs in the Lax formulation of the Calogero-Moser model, where one identifies

$$
\left\{r_{12}, L_{3}\right\}=\sum_{s=1}^{n} h_{s}^{(3)} \frac{d}{d q_{s}} r_{12},
$$

where $h_{s}, s=1, \ldots, n$ is a representation of a Cartan subalgebra ${ }^{1}$.

Note that the second one occurs in the Lax formulation of the Ruijsenaars-Schneider model [28] and is very much related to the first one as

$$
\left\{r_{12}, L_{3}\right\}=\sum_{s=1}^{n} h_{s}^{(3)} L_{3} \frac{d}{d q_{s}} r_{12} .
$$

Equation (4) now allows to identify the generic dYB equation (3) with a specific equation known as the classical Gervais-Neveu-Felder equation [10, 16, 18]. The problem of realizing an explicit algebraic form for the dynamical terms remains however open in many interesting cases.

It is crucial to underline here that in the Lax representation (1) there exists a one-to-one algebraic correspondence, parametrized by the linear $r$ matrix, between the Hamiltonian $H(L)$ triggering a specific time evolution, and the associated $M$ matrix. Namely [29] the $M$ matrix is given by

$$
M=\operatorname{Tr}_{1}\left(r_{12} d H(L)_{2}\right) .
$$

The Lax matrix $L$ however is characteristic of the whole Hamiltonian hierarchy and remains therefore unique.

\subsection{Hierarchy of Poisson brackets: Magri construction}

A dual formulation of Liouville integrability was proposed by Magri [21] where the hierarchy of Hamiltonians $H^{(k)}=\operatorname{Tr} L^{k}, k=1, \ldots, n$ acting simultaneously on the dynamical variables $p_{i}, q_{j}$ through a single Poisson bracket structure (e.g. the canonical one $\left\{p_{i}, q_{j}\right\}=\delta_{i j}$ ), is substituted by a hierarchy of mutually compatible Poisson brackets \{\}$_{(i)}$, where $i=1$ corresponds to the above canonical "first" bracket. The duality between the two formulations is summarized by the identity

$$
\left\{H^{(k)}, X(p, q)\right\}_{(l)}=\left\{H^{\left(k^{\prime}\right)}, X(p, q)\right\}_{\left(l^{\prime}\right)} \quad \text { for } \quad k+l=k^{\prime}+l^{\prime} .
$$

The explicit construction of the higher $(l \geq 2)$ Poisson brackets uses the so-called "recursion operator" (see, e.g., [25]).

It then follows that the Lax matrix for a given classically Liouville integrable system exhibits a corresponding hierarchy of $r$-matrix structures associated to each Poisson bracket in the Magri hierarchy. Such structures are explicitly known when the $r$-matrix for the first (canonical)

\footnotetext{
${ }^{1}$ In a more general, abstract context the Abelian property is dropped and one is lead to consider "non-Abelian dynamical algebras" [32].
} 
bracket is non-dynamical. For instance the second Poisson bracket is the famous quadratic Sklyanin bracket $[20,22,30]$ :

$$
\left\{L_{1}, L_{2}\right\}=\left[a_{12}, L_{1} L_{2}\right]+L_{1} s_{12} L_{2}-L_{2} s_{12} L_{1},
$$

where $a$ and $s$ are respectively the skew-symmetric, $a_{12}=\frac{1}{2}\left(r_{12}-r_{21}\right)$, and symmetric, $s_{12}=$ $\frac{1}{2}\left(r_{12}+r_{21}\right)$, part of the $r$-matrix. The Sklyanin bracket, properly said, corresponds to the case $s=0$.

Note that a quadratic Poisson bracket (5) takes in any case the general linear form (2) with a linear $r$ matrix defined as

$$
r_{12} \equiv \frac{1}{2}\left(a_{12} L_{2}+L_{2} a_{12}\right)-L_{2} s_{12}
$$

The third bracket has a more complicated form, cubic in terms of $L$, derived explicitly in [20].

\subsection{The problem of $r$-matrix construction}

Our purpose here is to give an explicit $r$-matrix structure for the second Poisson bracket of the rational $N$-site Calogero-Moser Lax matrix. This second Poisson structure was recently derived explicitly $[11,21]$ which makes it possible in principle to define an associated $r$-matrix structure. Our aim here is therefore to provide what we believe to be the first example of $r$ matrix structure for a second Poisson structure when the first Poisson structure is parametrized by a dynamical $r$ matrix [7] in a linear formulation (2). Indeed no explicit Sklyanin or Li-Parmentier formulation exists in such a case and we would thus provide or disprove the existence of such a formulation in this generic case.

The Poisson bracket hierarchy however is only formulated in terms of the adjoint invariants of the Lax matrix $I_{k}=\operatorname{Tr} L^{k}$ and mixed Lax-position matrix $J_{k}=\operatorname{Tr} L^{k} Q$ where $Q$ is the diagonal position matrix $\operatorname{Diag}\left(q_{1}, \ldots, q_{N}\right)$. Inverting this form to rexpress the Poisson brackets of the original first-bracket canonical variables (in which the Lax matrix elements have simple expressions) is our first aim, partially achieved in Section 3.

General remarks on $r$-matrix formulations are required here before we get into more details on our specific approach. It has been established [9] that given any finite-dimensional Lax matrix $L$ together with a Poisson structure guaranteeing Poisson commutation of the invariant traces or equivalently the eigenvalues, one may construct a linear $r$-matrix formulation (2) for the Poisson brackets of the components of $L$. This $r$-matrix is inherently non-skew symmetric and dynamical [29]. It is clear from example (6) and, more subtly, from the example of the Ruijsenaars-Schneider Lax matrix [31], that the linear $r$-matrix formulation à la BabelonViallet [9] has a priori no reason to be the most relevant or the most adapted formulation of a given Lax $r$-matrix structure, given in particular the algebro-geometric context of its construction. There is actually no general rule to indicate whether a linear, quadratic or even higher-level $r$-matrix formulation is required, although the above mentioned algebro-geometric context may be helpful in this respect.

Getting back to our specific issue, it appears here that although, as indicated above, one may not a priori expect an exact Li-Parmentier procedure, we should nevertheless assume a quadratic form such as (5) with arbitrary structure coefficients $a, b, c, d$. In view of the formal resemblance between the second Poisson structure on the unreduced phase space yielding the Calogero-Moser model [11] and the first Poisson structure on the unreduced phase space $G \times g$ yielding the rational Ruijsenaars Schneider model (see, e.g., discussion in [1]), this assumption seems all the more justified. It immediately eliminates a direct derivation à la Babelon-Viallet which would only yield a cumbersome linear $r$-matrix structure from which one would have to disentangle the $L$ dependance to get the quadratic coefficients $a, b, c, d$. 
Another way to derive $r$-matrix structures when the dynamical system is obtained by either a Hamiltonian reduction (first Poisson structure of Calogero-Moser, see [26]) or more generally (our problem here, see [11]) a projection on reduced symplectic leaves, is to follow algebraically the reduction of the $r$-matrix structure along the fibers of the symplectic reduction. It is certainly in principle the most significant and illuminating way to understand the "lower" $r$ matrix structure once derived, and has been achieved on a number of examples [4, 15, 27]. It is however a long and delicate procedure which may not be the best way for a small-sized Lax matrix. We have therefore decided to use a direct resolution of the consistency equations obtained by inserting the explicit Poisson brackets on the l.h.s. of (5).

\subsection{Outline of the derivation}

To obtain the Poisson brackets between physical variables $p, q$ we first of all (Section 2) derive some general Poisson bracket identities valid for all values of $N$, which allows us to reobtain the full $N=2$ second Poisson bracket structure derived in [11,21] and construct explicitly the $N=3$ Poisson brackets (Section 3). Formulae for $N=3$ are considerably more complicated and do not suggest at this time an obvious generalization to any value of $N \geq 4$.

We then (Section 4) propose a completely explicit form for second Poisson bracket of the $N=2$ site Lax matrix. This structure is defined as an explicit quadratic Sklyanin form (5) in terms of the Lax matrix $L$. It is identified with a representation of the classical limit of the second dynamical boundary algebra, recently built in [6]. It therefore does not match with the $r$-matrix formulation of the first Poisson structure of Calogero-Moser Lax matrix, which is in fact given by a representation of another dynamical boundary algebra, viz. the semi-dynamical boundary algebra of [3]. A direct Li-Parmentier type procedure for dynamical $r$-matrices is therefore invalidated by this example.

The case $N=3$ seems at this time too complex and not clearly enough understood to allow for a reasonable attempt at building an $r$-matrix structure. We conclude with some remarks and proposals.

\section{General properties of $N$-Calogero}

The Lax matrix and position matrix for the rational $A_{n}$ Calogero-Moser model are defined as

$$
L=\left(\begin{array}{cccc}
p_{1} & \frac{1}{q_{12}} & \ldots & \frac{1}{q_{1 N}} \\
\frac{-1}{q_{12}} & p_{2} & \ddots & \vdots \\
\vdots & \ddots & \ddots & \frac{1}{q_{N-1, N}} \\
\frac{-1}{q_{1 N}} & \cdots & \frac{-1}{q_{N-1, N}} & p_{N}
\end{array}\right) \quad \text { and } \quad Q=\left(\begin{array}{cccc}
q_{1} & 0 & \ldots & 0 \\
0 & q_{2} & \ddots & \vdots \\
\vdots & \ddots & \ddots & 0 \\
0 & \ldots & 0 & q_{N}
\end{array}\right) \text {, }
$$

where $q_{i j}=q_{i}-q_{j}$.

The second Poisson structure is expressed [11] in the basis

$$
I_{n}=\frac{1}{n} \operatorname{Tr} L^{n} \quad \text { and } \quad J_{n+1}=\operatorname{Tr}\left(Q L^{n}\right) .
$$

It reads

$$
\left\{I_{n}, I_{m}\right\}=0, \quad\left\{J_{n}, I_{m}\right\}=(m+n-1) I_{m+n-1}, \quad\left\{J_{n}, J_{m}\right\}=(n-m) J_{m+n-1} .
$$

We call this Poisson bracket algebra $\mathcal{A}_{N}$. Remark that the first generators $I_{1}$ and $J_{1}$ correspond to the center of mass position and momentum,

$$
p_{0}=I_{1}=\sum_{j=1}^{N} p_{j} \quad \text { and } \quad q_{0}=J_{1}=\sum_{j=1}^{N} q_{j},
$$


while $I_{2}$ is the Calogero Hamiltonian

$$
H=\sum_{j=1}^{N} p_{j}^{2}-2 \sum_{j \neq i}^{N} \frac{1}{\left(q_{i}-q_{j}\right)^{2}}
$$

\subsection{Decoupling of the center of mass position}

We extract consistently the centre-of-mass variable $q_{0} \equiv J_{1}$ by setting

$$
\widetilde{Q}=Q-\frac{\operatorname{Tr}(Q)}{N} \mathbb{I}=Q-\frac{q_{0}}{N} \mathbb{I} \quad \text { and } \quad \widetilde{J}_{n+1}=\operatorname{Tr}\left(\widetilde{Q} I_{n}\right)=J_{n+1}-\frac{n}{N} q_{0} I_{n}
$$

with the convention $\lim _{n \rightarrow 0}\left(n I_{n}\right)=N$.

Proposition 1. The algebra $\mathcal{A}_{N}$ is the semi-direct sum of the subalgebra $\widetilde{\mathcal{A}}_{N}$, generated by $I_{n}$ and $\widetilde{J}_{n}$ and the $(P B-)$ commutative algebra $\left\{q_{0}\right\}$

$$
\begin{aligned}
& \left\{I_{n}, I_{m}\right\}=0, \quad\left\{\widetilde{J}_{n+1}, I_{m}\right\}=(m+n) I_{m+n}-\frac{m n}{N} I_{m} I_{n}, \\
& \left\{\widetilde{J}_{n+1}, \widetilde{J}_{m+1}\right\}=(n-m) \widetilde{J}_{m+n+1}+\frac{m n}{N}\left(\widetilde{J}_{m+1} I_{n}-\widetilde{J}_{n+1} I_{m}\right), \\
& \left\{q_{0}, I_{m}\right\}=m I_{m}, \quad\left\{q_{0}, \widetilde{J}_{n+1}\right\}=n \widetilde{J}_{n+1} .
\end{aligned}
$$

The structure of the algebra $\widetilde{\mathcal{A}}_{N}$ is entirely determined by the PBs of the $p_{i}$ 's and the $q_{i j}$ 's and does not depend on the PBs of $q_{0}$.

Proof. The PBs are obtained by direct calculation. It follows that, since $\widetilde{Q}$ and $L$ do not depend on $q_{0}$, the PBs of $q_{0}$ with $I$ and $J$ variables are not relevant in the calculation of the $L$ Poisson brackets and the associated $r$-matrix structure. They shall be considered separately.

\subsection{PBs of the center of mass momentum}

Proposition 2. The Poisson brackets of $p_{0}$ are given by

$$
\left\{p_{0}, q_{j}\right\}=-p_{j} \quad \text { and } \quad\left\{p_{0}, p_{j}\right\}=-2 \sum_{n \neq j} q_{j n}^{-3} .
$$

Proof. Let

$$
K=\left(\begin{array}{cccc}
a_{1} & q_{12}^{-2} & \ldots & q_{1 N}^{-2} \\
q_{12}^{-2} & a_{2} & \ddots & \vdots \\
\vdots & \ddots & \ddots & q_{N-1, N}^{-2} \\
q_{1 N}^{-2} & \cdots & q_{N-1, N}^{-2} & a_{N}
\end{array}\right) \quad \text { with } \quad a_{j}=-\sum_{n \neq j} q_{n j}^{-2}
$$

then (8) is equivalent to

$$
\left\{p_{0}, L\right\}=[L, K] \quad \text { and } \quad\left\{p_{0}, Q\right\}=[Q, K]-L .
$$

This matricial form of the PBs implies

$$
\left\{p_{0}, I_{n}\right\}=\sum_{m=0}^{n} \operatorname{Tr} L^{m}[L, K] L^{n-m-1}=\operatorname{Tr}\left(L^{n-1} K-K L^{n-1}\right)=0
$$


and

$$
\begin{aligned}
\left\{p_{0}, J_{n+1}\right\} & =\operatorname{Tr}([Q, K]-L) L^{n}+\sum_{m=0}^{n+1} \operatorname{Tr} Q L^{m}[L, K] L^{n-m} \\
& =\operatorname{Tr}\left(K Q L^{n}-Q L^{n} K\right)-\operatorname{Tr} L^{n+1}=-(n+1) I_{n+1} .
\end{aligned}
$$

Hence, (8) reproduce the PBs of $p_{0}$ with all the generators of $\mathcal{A}_{N}$. Since the correspondence between $I, J$ and $p, q$ variables is one-to-one [11], the PB's of $p_{0}$ with $q_{i}, p_{j}$ are univocally determined by the PB's of $p_{0}$ with $I_{i}, J_{j}$ establishing that (8) is correct.

It is interesting to remark that this matrix $K$ (used above) is such that the sum of elements on every line or every column yields 0 . It implies that $K$ commutes with the matrix $\mu \equiv \sum_{i \neq j} e_{i j}$ which is the moment map used to define the Calogero-Moser model by Hamiltonian reduction of a free motion on the cotangent bundle of the Lie algebra $M_{n}(\mathbb{C})$ [26]. However the corresponding Poisson structure is the first, not second one and the meaning of this property of $K$ is therefore not clear.

Corollary 1. For any function of $\vec{q}$ and $\vec{p}$, we have

$$
\left\{p_{0}, f(\vec{q}, \vec{p})\right\}=\mathcal{D} f(\vec{q}, \vec{p}) \quad \text { with } \quad \mathcal{D}=\sum_{n=1}^{N}\left\{p_{n} \frac{\partial}{\partial q_{n}}-2 \sum_{j \neq n} q_{j n}^{-3} \frac{\partial}{\partial q_{j}}\right\} .
$$

Proof. Direct calculation using (8).

\section{Second Calogero-Poisson brackets}

\section{1 $\quad N=2$ Poisson brackets}

These second PBs have been already calculated in [11]. We recall them for the sake of completeness and show on a simple case the method we use for $N=3$.

From the calculation of Section 2.2, one deduces that

$$
\left\{p_{1}, p_{2}\right\}=-\frac{1}{q_{12}^{3}} \quad \text { and } \quad\left\{p_{j}, q_{k}\right\}=-\delta_{j k} p_{j}+z_{j k} \quad \text { with } \quad z_{1 j}+z_{2 j}=0 \quad \forall j,
$$

which leaves us with three unknowns, $z_{11}, z_{22}$ and the $\mathrm{PB}\left\{q_{1}, q_{2}\right\}$.

Plugging this partial result into the PBs given in (7), one deduce the final form, parametrized as

$$
\begin{aligned}
& z_{11}=-z_{22}=-z_{21}=z_{12}=\frac{\left(p_{1}-p_{2}\right)}{\left(q_{1}-q_{2}\right)^{2}} \frac{1}{\frac{4}{\left(q_{1}-q_{2}\right)^{2}}-\left(p_{1}-p_{2}\right)^{2}}, \\
& \left\{q_{1}, q_{2}\right\}=\frac{1}{\left(q_{1}-q_{2}\right)} \frac{4}{\frac{4}{\left(q_{1}-q_{2}\right)^{2}}-\left(p_{1}-p_{2}\right)^{2}} .
\end{aligned}
$$

As already mentioned, these expressions were calculated in [11], directly from the PBs of the $I_{n}$ 's and $J_{m}$ 's. However, a direct calculation becomes highly complicated for larger values of $N$, and one needs to use the results obtained in Sections 2.1 and 2.2. This defines the strategy we will adopt in the next subsection. 


\section{$3.2 N=3$ Calogero}

We set the following forms for the $\{p, p\}$ and $\{p, q\}$ brackets

$$
\begin{aligned}
& \left\{p_{i}, p_{j}\right\}=-\frac{2}{q_{i j}^{3}}+x_{i j} \quad \text { and } \quad\left\{p_{i}, q_{j k}\right\}=\left(\delta_{i k}-\delta_{j i}\right) p_{i}+z_{i ; j k}, \\
& \text { with } \quad x_{i j}=-x_{j i} \quad \text { and } \quad z_{i ; j k}=-z_{i ; k j} .
\end{aligned}
$$

A careful study of Jacobi identity for the triplet $p_{0}, p_{1}, p_{2}$ finally yields

$$
x_{12}=x_{23}=x_{31} \equiv x_{0} .
$$

Consistency conditions on the $z$ coefficients read

$$
z_{1 ; j k}+z_{2 ; j k}+z_{3 ; j k}=0 \quad \forall j \neq k, \quad z_{i ; 12}+z_{i ; 23}+z_{i ; 31}=0 \quad \forall i .
$$

Explicit resolution of the Poisson bracket structure can then be achieved and yields

$$
z_{i ; j k}=-\left(\frac{q_{j k}}{q_{i i_{2}} q_{i i_{3}}}\right)^{3} \frac{n_{i ; j k}}{\mathrm{~d}} \quad \text { with } \quad\left(i, i_{2}, i_{3}\right)=\text { circ.perm. }(1,2,3) .
$$

Two forms of $n$ coefficients are defined depending on which independent indices are present. One gets

$$
\begin{aligned}
n_{i ; j k}= & -q_{i j} q_{i k}\left\{q_{i j}^{2} q_{i k}^{2}\left(-q_{i j}^{3} p_{j}^{2}+q_{i k}^{3} p_{k}^{2}\right)+p_{i}\left(p_{j}-p_{k}\right) q_{i j}^{2} q_{i k}^{2}\left(q_{i j}+\frac{1}{2} q_{i k}\right) \boldsymbol{q}^{2}\right. \\
& \left.-p_{j} p_{k} q_{i j}^{2} q_{i k}^{2} q_{j k}\left(q_{j k}^{2}+3 q_{i k} q_{i j}\right)+\frac{1}{2} \boldsymbol{q}^{2} q_{j k}\left(q_{i j}+q_{i k}\right)^{2}\right\} x_{0}-\boldsymbol{q}^{2}\left(q_{i j}+q_{i k}\right)^{2}, \\
n_{i ; i j}= & \left\{-\left(q_{i j} q_{j k} q_{k i}\right)^{3}\left(p_{j}-p_{i}\right)\left(p_{j}-p_{k}\right)+\frac{1}{2} \boldsymbol{q}^{2} q_{i j} q_{i k} q_{j k}\left(q_{i j}+q_{i k}\right)\left(q_{i k}+q_{j k}\right)\right\} x_{0} \\
& +4 q_{i k}^{3}-2 q_{i j} q_{j k}\left(q_{i k}^{2}+q_{i j} q_{j k}\right) .
\end{aligned}
$$

Here it is understood that $(i, j, k)$ is any permutation of the three indices $1,2,3$. In addition one defines

$$
\mathrm{d}=-\boldsymbol{q}^{2}\left(p_{1}\left(q_{12}+q_{13}\right)+p_{2}\left(q_{23}+q_{21}\right)+p_{3}\left(q_{31}+q_{32}\right)\right), \quad \boldsymbol{q}^{2}=q_{12}^{2}+q_{23}^{2}+q_{13}^{2} .
$$

Finally the single, pure $q_{i j}$ bracket reads

$$
\begin{aligned}
\left\{q_{12}, q_{23}\right\} & =-\frac{n_{12} n_{23}}{2 \mathrm{~d}} \\
n_{12} n_{23}= & q_{12} q_{13} q_{23} x_{0}\left\{\left(q_{12} q_{13} q_{23}\right)^{2}\left(p_{1} p_{2}\left(p_{1}-p_{2}\right)+p_{2} p_{3}\left(p_{2}-p_{3}\right)+p_{1} p_{3}\left(p_{1}-p_{3}\right)\right)\right. \\
& \left.+q_{23}^{2}\left(q_{12}-q_{31}\right) p_{1}-q_{13}^{2}\left(q_{23}-q_{12}\right) p_{2}-q_{12}^{2}\left(q_{13}+q_{23}\right) p_{3}\right\} \\
& +\boldsymbol{q}^{2}\left\{p_{1} q_{23}\left(q_{12}+q_{13}\right)+p_{2} q_{13}\left(q_{12}+q_{32}\right)-p_{3} q_{12}\left(q_{13}+q_{23}\right)\right\} .
\end{aligned}
$$

The quantity $x_{0}$ is explicitly obtained as a very complicated rational function of all dynamical variables $p$ and $q_{i}-q_{j}$. It can be characterized however as the single solution of the differential equation $(\mathcal{D}$ being defined in Corollary 1$)$

$$
\begin{aligned}
\mathcal{D} x_{0}= & \frac{x_{0}}{q_{12} q_{23} q_{31} \mathrm{~d}}\left\{q_{23}\left(q_{12}^{4}+q_{13}^{4}\right) p_{1}^{2}+q_{31}\left(q_{21}^{4}+q_{23}^{4}\right) p_{2}^{2}+q_{12}\left(q_{31}^{4}+q_{31}^{4}\right) p_{3}^{2}\right. \\
& +p_{2} p_{3} q_{23}\left(2 q_{23}^{4}+3 q_{12} q_{23}^{2} q_{13}-q_{12}^{2} q_{13}^{2}\right)+p_{1} p_{2} q_{12}\left(2 q_{12}^{4}+3 q_{23} q_{12}^{2} q_{13}-q_{23}^{2} q_{13}^{2}\right)
\end{aligned}
$$




$$
\begin{aligned}
& +p_{1} p_{3} q_{31}\left(2 q_{13}^{4}-3 q_{23} q_{13}^{2} q_{12}-q_{23}^{2} q_{12}^{2}\right)+\frac{\left(\boldsymbol{q}^{2}\right)^{3}}{4 q_{12} q_{23} q_{31}} \\
& +\frac{2\left(q_{13}^{6}+q_{12}^{6}+q_{23}^{6}\right)-6 q_{23}^{2} q_{12}^{2} q_{13}^{2}}{\left(q_{12} q_{23} q_{31}\right)^{3} \mathrm{~d}}
\end{aligned}
$$

with the particular value at $q_{12}=q_{23}$

$$
\left.x_{0}\right|_{q_{12}=q_{23}}=-18 \frac{81-3 q_{12}^{2}\left(p_{3}+p_{1}-2 p_{2}\right)^{2}-4 q_{12}^{4}\left(p_{2}-p_{1}\right)\left(p_{1}-p_{3}\right)^{2}\left(p_{3}-p_{2}\right)}{q_{13} q_{12}^{2} g\left(q_{12}\right) g\left(-q_{12}\right)}
$$

with

$$
\begin{aligned}
g\left(q_{12}\right)= & 27+27 q_{12}\left(p_{3}-p_{1}\right)+4 q_{12}^{2}\left(2 p_{3}-p_{2}-p_{1}\right)\left(p_{2}+p_{3}-2 p_{1}\right) \\
& +4 q_{12}^{3}\left(p_{2}-p_{1}\right)\left(p_{3}-p_{1}\right)\left(p_{3}-p_{2}\right) .
\end{aligned}
$$

Note also that

$$
x_{0} \sim \frac{-2}{q_{23} q_{13} q_{12}} \quad \text { for } \quad q_{12} \rightarrow 0
$$

for $q_{23}$ finite.

\subsection{Particular cases}

We present here some particular cases where the PBs simplify drastically. They correspond to particular positions of the particles. Remark that in some cases, this choice of particular positions make the Lax formalism ill-defined, but the PBs are themselves well-defined.

\subsubsection{Three free particles}

If we consider $q_{12}=q_{23} \rightarrow \infty$, the three particles are far away one from each other, so that they can be considered as decoupled. Indeed, in that case, the PBs simplify to

$$
\left\{q_{i}, q_{j}\right\}=0, \quad\left\{p_{i}, q_{j}\right\}=-\delta_{i j} p_{i}, \quad\left\{p_{i}, p_{j}\right\}=-\frac{2}{q_{i j}^{3}} .
$$

One recognizes the second $\mathrm{PB}$ structure of free particles.

Let us note that in order to take properly the limit, one has to explicitly use the behavior

$$
x_{0} \sim \frac{9}{4\left(p_{2}-p_{1}\right)\left(p_{2}-p_{3}\right) q_{12}^{5}} \quad \text { when } \quad q_{23}=q_{12} \rightarrow \infty .
$$

\subsubsection{One free particle}

If we now consider that only one particle, say particle 1, is far from the two others, one has to take the limit $q_{12} \rightarrow \infty$ keeping $q_{23}$ finite. The first particle (associated to the index 1) decouples while the particles 2 and 3 still interact. Indeed, in this case, the PBs simplify to

$$
\begin{aligned}
& \left\{q_{1}, q_{j}\right\}=0, \quad\left\{p_{j}, q_{1}\right\}=-\delta_{1 j} p_{1}, \quad\left\{p_{1}, q_{j}\right\}=-\delta_{1 j} p_{1}, \quad\left\{p_{1}, p_{j}\right\}=-\frac{2}{q_{1 j}^{3}} \quad \forall j, \\
& \left\{q_{2}, q_{3}\right\}=\frac{-2 q_{23}}{q_{23}^{2}\left(p_{2}-p_{3}\right)^{2}-4}, \quad\left\{p_{2}, p_{3}\right\}=-\frac{2}{q_{23}^{3}}, \\
& \left\{p_{j}, q_{k}\right\}=-\delta_{k j} p_{j}+z_{k j}, \quad j, k=2,3, \\
& z_{2 ; 12}=-\frac{p_{2}-p_{3}}{q_{23}^{2}\left(p_{2}-p_{3}\right)^{2}-4}, \quad z_{1 ; 12}=0, \quad z_{1 ; 23}=0,
\end{aligned}
$$




$$
\left\{q_{12}, q_{23}\right\}=-\frac{2 q_{23}}{q_{23}^{2}\left(p_{2}-p_{3}\right)^{2}-4} .
$$

One recovers indeed $z_{j k}$ such as computed in (9). Again, one needs to know that for $q_{12} \rightarrow \infty$,

$$
x_{0} \sim \frac{2}{q_{23}^{2}\left(p_{1}^{2}-p_{1}\left(p_{3}-p_{2}\right)+p_{2} p_{3}\right)+1}\left(\frac{-1 / q_{23}}{\left(q_{23}+q_{12}\right) q_{12}}+\frac{\left(p_{3}-p_{2}\right)\left(2 p_{1}-p_{3}-p_{2}\right) q_{23}^{2}}{\left(q_{23}^{2}\left(p_{3}-p_{2}\right)^{2}-4\right)\left(q_{23}+q_{12}\right) q_{12}^{2}}\right)
$$

to get a correct answer.

\section{Dynamical $r$-matrix algebra for $N=2$ Calogero}

\subsection{The quadratic algebra}

Let us now formulate the Poisson bracket structure (9) in terms of an $r$-matrix structure. We postulate that a quadratic formulation mimicking (5) will be adequate for this second Poisson bracket, although both $a$ and $s$ matrices will be expected to be dynamical. We recall that given a classical Lax matrix $\ell$, the most general quadratic form for the associated Poisson structure is

$$
\left\{\ell_{1}, \ell_{2}\right\}=a_{12} \ell_{1} \ell_{2}+\ell_{1} b_{12} \ell_{2}-\ell_{2} c_{12} \ell_{1}-\ell_{1} \ell_{2} d_{12}
$$

where consistency conditions imply that $a_{12}=-a_{21}, d_{12}=-d_{21}, b_{12}=c_{21}$. Note that (10) implies that the functions $\left\{\operatorname{tr} \ell^{m}, m \in \mathbb{Z}_{+}\right\}$Poisson-commute if $a+b=c+d$. A more general trace formula, $\operatorname{tr}\left(\gamma^{-1} \ell\right)^{m}$, occurs whenever a scalar matrix $\gamma$ exists such that

$$
a_{12} \gamma_{1} \gamma_{2}+\gamma_{1} b_{12} \gamma_{2}-\gamma_{2} c_{12} \gamma_{1}-\gamma_{1} \gamma_{2} d_{12}=0
$$

see [17].

Dynamical dependence of $a b c d$ now is assumed to be solely on coordinates $q_{i}, i=1, \ldots, n$, on a dual $\mathfrak{h}^{*}$ of the Cartan subalgebra $\mathfrak{h}$ in $\operatorname{sl}(n, \mathbb{C})$.

In the 2 sites case we get

$$
\ell=\left(\begin{array}{cc}
p_{1} & \frac{1}{q_{12}} \\
\frac{-1}{q_{12}} & p_{2}
\end{array}\right)
$$

The PB deduced from (9) reads

$$
\left\{\ell_{1}, \ell_{2}\right\}=\frac{1}{q_{12}}\left(\begin{array}{cccc}
0 & \frac{p_{1}}{q_{12}} & -\frac{p_{1}}{q_{12}} & 0 \\
-\frac{p_{1}}{q_{12}} & -\frac{2}{\left(q_{12}\right)^{2}} & 0 & \frac{p_{2}}{q_{12}} \\
\frac{p_{1}}{q_{12}} & 0 & \frac{2}{\left(q_{12}\right)^{2}} & -\frac{p_{2}}{q_{12}} \\
0 & -\frac{p_{2}}{q_{12}} & \frac{p_{2}}{q_{12}} & 0
\end{array}\right) .
$$

As in the linear case (3), (4), associativity for the PB structure (10) is implied by algebraic consistency conditions (Yang-Baxter classical equations) for $a, b, c, d$, provided the a priori undetermined bracket $\left\{r_{12}, \ell_{3}\right\}, r=a, b, c, d$, be of an algebraic form. We postulate here the following form for this $\mathrm{PB}$

$$
\left\{r_{12}, \ell_{3}\right\}=\epsilon_{R}\left(h_{3} \partial r_{12}\right) \ell_{3}+\epsilon_{L} \ell_{3} h_{3} \partial r_{12},
$$




$$
h \partial=\sum_{i=1}^{n} \mu e_{i i} \otimes \frac{\partial}{\partial q_{i}},
$$

where $e_{i i} \in \mathfrak{h}, \epsilon_{R}, \epsilon_{L}$ are $c$-numbers to be determined. Notice the difference in homogeneity factors in $l$ with respect to the linear case (4).

We will see below that this postulate is consistent and the correct choice of parameters $\epsilon$ is

$$
\epsilon_{L}=\epsilon_{R}=\frac{1}{2}
$$

A solution to express the $\mathrm{PB}(11)$ as a quadratic form is given by

$$
a_{12}=d_{12}=\left(\begin{array}{cccc}
0 & 0 & 0 & 0 \\
0 & \frac{-w_{1}}{2 q_{12}} & \frac{1}{2 q_{12}} & 0 \\
0 & \frac{-1}{2 q_{12}} & \frac{w_{1}}{2 q_{12}} & 0 \\
0 & 0 & 0 & 0
\end{array}\right), \quad b_{12}=c_{21}=c_{12}=\left(\begin{array}{cccc}
\frac{-w_{1}}{2 q_{12}} & 0 & 0 & \frac{1}{2 q_{12}} \\
0 & 0 & 0 & 0 \\
0 & 0 & 0 & 0 \\
\frac{-1}{2 q_{12}} & 0 & 0 & \frac{w_{1}}{2 q_{12}}
\end{array}\right) \text {, }
$$

where $w_{1}$ is a free parameter.

Jacobi identity for the quadratic PB is then ensured, given (12) by the following classical dynamical Yang-Baxter equations

$$
\begin{aligned}
& {\left[a_{12}, a_{13}\right]+\left[a_{12}, a_{23}\right]+\left[a_{32}, a_{13}\right]+\frac{1}{2}\left(h_{3} \partial a_{12}+h_{1} \partial a_{23}+h_{2} \partial a_{31}\right)=0,} \\
& {\left[d_{12}, d_{13}\right]+\left[d_{12}, d_{23}\right]+\left[d_{32}, d_{13}\right]+\frac{1}{2}\left(h_{3} \partial d_{12}+h_{1} \partial d_{23}+h_{2} \partial d_{31}\right)=0,} \\
& {\left[a_{12}, c_{13}+c_{23}\right]+\left[c_{13}, c_{23}\right]+\frac{1}{2}\left(-h_{3} \partial a_{12}+h_{1} \partial c_{23}-h_{2} \partial c_{13}\right)=0,} \\
& {\left[d_{12}, b_{13}+b_{23}\right]+\left[b_{13}, b_{23}\right]+\frac{1}{2}\left(-h_{3} \partial d_{12}+h_{1} \partial b_{23}-h_{2} \partial b_{13}\right)=0 .}
\end{aligned}
$$

In the absence of dynamical term, one would recover the usual classical quadratic algebra [17].

A connection between the classical DYB equations associated with quadratic and linear Poisson brackets is established as follows: In the simplest case of Poisson commutation of traces when $a+b=c+d$ in (10) the matrix $r \equiv a+b$ obeys the linear dynamical Yang-Baxter equation obtained from (3), (4), that is, with two derivative terms provided that $a, b, c, d$ obey (14)-(17), coupled DYB with three derivative terms.

This result however does not imply that (3) and (10) are identified as consistent first and second Poisson structure for $l$ since the algebraic forms (14)-(17) require in addition respectively the identification of the Poisson brackets of $l$ with $r$ or $a, b, c, d$ as (4) or (12) which is not implied in any way by the form of PB's (3) and (10). In other words, contrary to the non-dynamical case (Sklyanin bracket) one cannot establish that the quadratic form (10) be a consistent Poisson structure solely from the fact that (3) be one such structure even if (14)-(17) hold. One then immediately observes that (14)-(17) is a classical limit $(\hbar \rightarrow 0)$ of a set of 4 dynamical YangBaxter equations first formulated in [6]

$$
\begin{aligned}
& A_{12}(\boldsymbol{q}) A_{13}\left(\boldsymbol{q}-\epsilon_{R} h^{(2)}\right) A_{23}(\boldsymbol{q})=A_{23}\left(\boldsymbol{q}-\epsilon_{R} h^{(1)}\right) A_{13}(\boldsymbol{q}) A_{12}\left(\boldsymbol{q}-\epsilon_{R} h^{(3)}\right), \\
& D_{12}\left(\boldsymbol{q}+\epsilon_{L} h^{(3)}\right) D_{13}(\boldsymbol{q}) D_{23}\left(\boldsymbol{q}+\epsilon_{L} h^{(1)}\right)=D_{23}(\boldsymbol{q}) D_{13}\left(\boldsymbol{q}+\epsilon_{L} h^{(2)}\right) D_{12}(\boldsymbol{q}), \\
& A_{12}(\boldsymbol{q}) C_{13}\left(\boldsymbol{q}-\epsilon_{R} h^{(2)}\right) C_{23}(\boldsymbol{q})=C_{23}\left(\boldsymbol{q}-\epsilon_{R} h^{(1)}\right) C_{13}(\boldsymbol{q}) A_{12}\left(\boldsymbol{q}+\epsilon_{L} h^{(3)}\right), \\
& D_{12}\left(\boldsymbol{q}-\epsilon_{R} h^{(3)}\right) B_{13}(\boldsymbol{q}) B_{23}\left(\boldsymbol{q}+\epsilon_{L} h^{(1)}\right)=B_{23}(\boldsymbol{q}) B_{13}\left(\boldsymbol{q}+\epsilon_{L} h^{(2)}\right) D_{12}(\boldsymbol{q}),
\end{aligned}
$$


with the particular choice of "weight parameters" $\epsilon_{L}=\epsilon_{R}=\frac{1}{2}$. The classical limit is defined by setting

$$
\begin{aligned}
& R(\boldsymbol{q})=\mathbb{I}+\hbar r(\boldsymbol{q})+o\left(\hbar^{2}\right), \quad R=A, B, C, D \quad \text { and } \quad r=a, b, c, d, \\
& h^{(i)}=\hbar h_{i}+o\left(\hbar^{3}\right),
\end{aligned}
$$

and keeping the order $\hbar^{2}$ in (18)-(21), orders 1 and $\hbar$ being trivial.

These 4 equations are in turn characterized as sufficient conditions for associativity of a quantum quadratic dynamical exchange algebra defined generically in [6]

$$
\begin{aligned}
& A_{12}(\boldsymbol{q}) K_{1}\left(\boldsymbol{q}-\epsilon_{R} h^{(2)}\right) B_{12}(\boldsymbol{q}) K_{2}\left(\boldsymbol{q}+\epsilon_{L} h^{(1)}\right) \\
& \quad=K_{2}\left(\boldsymbol{q}-\epsilon_{R} h^{(1)}\right) C_{12}(\boldsymbol{q}) K_{1}\left(\boldsymbol{q}+\epsilon_{L} h^{(2)}\right) D_{12}(\boldsymbol{q})
\end{aligned}
$$

assuming a set of zero-weight conditions

$$
\begin{aligned}
& \epsilon_{R}\left[h^{(1)}+h^{(2)}, A_{12}\right]=\epsilon_{L}\left[h^{(1)}+h^{(2)}, D_{12}\right]=0, \\
& {\left[\epsilon_{R} h^{(1)}-\epsilon_{L} h^{(2)}, C_{12}\right]=\left[\epsilon_{L} h^{(1)}-\epsilon_{R} h^{(2)}, B_{12}\right]=0,}
\end{aligned}
$$

and unitary hypothesis

$$
A_{12} A_{21}=D_{12} D_{21}=\mathbb{I} \otimes \mathbb{I}, \quad C_{12}=B_{21} .
$$

Altogether, these relations ensure associativity of the product in the dynamical algebra.

Note that the free parameter $w_{1}$ is the signature in this classical limit of one particular gauge covariance of the dynamical Yang-Baxter equation for $A$, pointed out, e.g., in [5] under which diagonal coordinates $d_{i j}$ of $R$-matrix on basis elements $e_{i j} \otimes e_{j i}$ in $M_{n}(\mathbb{C}) \otimes M_{n}(\mathbb{C})$ contain constant parameters $g_{i j} \equiv f_{i}-f_{j}$ and $f_{i}$ are arbitrary non-dynamical c-numbers. Here $\hbar w_{1} \equiv f_{1}-f_{2}$.

\subsection{Comparison with the first Poisson bracket $r$-matrix}

We recall that the first Poisson bracket of rational Calogero-Moser model is expressed linearly in terms of the Lax matrix $L$ following the formulation (2). The $r$-matrix takes the form

$$
r=\sum_{i \neq j} \frac{1}{q_{i}-q_{j}} e_{i j} \otimes e_{j i}+\sum_{k \neq j} \frac{1}{q_{k}-q_{j}} e_{k k} \otimes e_{k j} .
$$

It is interpreted $[2,3,24,31]$ as a combination $r_{12}=d_{12}+c_{12}$ of two matrices realizing with $b_{12} \equiv c_{21}$ and $a=d+c-b$ a classical semi-dynamical reflection algebra corresponding to the choice $\epsilon_{L}=0, \epsilon_{R}=1$ in (18)-(21). In particular the symmetric part of $r$ is now a sum $\frac{1}{2}(b+c)$ of two matrices with respective weights $(0,1)$ and $(1,0)$ under adjoint action of $\mathfrak{h} \oplus \mathfrak{h}$. It is therefore not related with the abcd quadruplet realizing the second Poisson structure of the rational CM Lax matrix, although the $d$ matrices themselves are identical. The second CM bracket is therefore not realized as a Li-Parmentier-type quadratization of the first CM bracket. This counterexample arising in the simplest available situation for dynamical $r$-matrices thus eliminates any possibility of extending directly the Sklyanin-Li-Parmentier procedure for higher Poisson brackets to the case of dynamical $r$-matrices.

Curiously enough a quadratic Poisson bracket involving the components $a, b, c, d$ deduced from the $r$-matrix of the first $\mathrm{CM}$ bracket does exist: it arises in the formulation of the first $\mathrm{PB}$ of the Ruijsenaars-Schneider rational Lax matrix [28, 31]. 


\section{Open questions}

The formulation of a simple algebraic relation à la Sklyanin between first and second Poisson bracket structure in the context of Lax matrices where dynamical $r$-matrices arise in the linear expression of their first PB seems thus, if not altogether excluded, at least unreachable at the moment. Other issues remain open at this time:

- Define the crossed $r$-matrix formulation for $L$ and $Q$, and the $r$-matrix structure for $Q$ when $N=2$. In this way a complete understanding of the second PB structure including the $\{I, J\}$ and $\{J, J\}$ brackets, will be achieved.

- Define the $r$-matrix formulation describing the second PB structure for $N=2$ RuijsenaarsSchneider model (starting with the rational case). In fact a form for this second PB structure has been conjectured [1] but not explicitly built in terms of Lax matrix (only in terms of trace invariants extending naturally the variables $I$ and $J$ ). One conjectures here that some cubic- $l$ dependent form will be relevant.

- Define the $r$-matrix formulation for $N=3$. The complexity of the expressions for the coordinate Poisson brackets seems to present a difficult technical challenge here. This technical complexity indicates in any case that the $r$-matrix quadruplet in a postulated quadratic form will exhibit a dependence on both $p$ and $q$ variables, suggesting that the dynamical dependance here goes beyond the Gervais-Neveu-Felder formulation (4). A similar issue arose some time ago [12] for $N \geq 4$ elliptic Calogero-Moser Lax formulation without spectral parameter, and has not been satisfactorily solved since. The issue of $p, q$ dependant $r$-matrices is in any case a yet mostly unexplored one which we hope to come back to in a near future.

\section{Acknowledgements}

This work was sponsored by CNRS, Université de Cergy-Pontoise, Université de Savoie and ANR Project DIADEMS (Programme Blanc ANR SIMI1 2010-BLAN-0120-02). J.A. wishes to thank LAPTh for their kind hospitality. We thank the referees for their helpful queries.

\section{References}

[1] Aniceto I., Avan J., Jevicki A., Poisson structures of Calogero-Moser and Ruijsenaars-Schneider models, J. Phys. A: Math. Gen. 43 (2010), 185201, 14 pages, arXiv:0912.3468.

[2] Arutyunov G.E., Chekhov L.O., Frolov S.A., R-matrix quantization of the elliptic Ruijsenaars-Schneider model, Comm. Math. Phys. 192 (1998), 405-432, q-alg/9612032.

[3] Arutyunov G.E., Frolov S.A., Quantum dynamical $R$-matrices and quantum Frobenius group, Comm. Math. Phys. 191 (1998), 15-29, q-alg/9610009.

[4] Avan J., Babelon O., Talon M., Construction of the classical R-matrices for the Toda and Calogero models, St. Petersburg Math. J. 6 (1994), 255-274, hep-th/9606102.

[5] Avan J., Billaud B., Rollet G., Classification of non-affine non-Hecke dynamical R-matrices, SIGMA 8 (2012), 064, 45 pages, arXiv:1204.2746.

[6] Avan J., Ragoucy E., A new dynamical reflection algebra and related quantum integrable systems, Lett. Math. Phys. 101 (2012), 85-101, arXiv:1106.3264.

[7] Avan J., Talon M., Classical R-matrix structure for the Calogero model, Phys. Lett. B 303 (1993), 33-37, hep-th/9210128.

[8] Babelon O., Bernard D., Talon M., Introduction to classical integrable systems, Cambridge Monographs on Mathematical Physics, Cambridge University Press, Cambridge, 2003.

[9] Babelon O., Viallet C.M., Hamiltonian structures and Lax equations, Phys. Lett. B 237 (1990), 411-416. 
[10] Balog J., Dạbrowski L., Fehér L., Classical r-matrix and exchange algebra in WZNW and Toda theories, Phys. Lett. B 244 (1990), 227-234.

[11] Bartocci C., Falqui G., Mencattini I., Ortenzi G., Pedroni M., On the geometric origin of the bi-Hamiltonian structure of the Calogero-Moser system, Int. Math. Res. Not. 2010 (2010), no. 2, 279-296, arXiv:0902.0953.

[12] Braden H.W., Suzuki T., R-matrices for elliptic Calogero-Moser models, Lett. Math. Phys. 30 (1994), 147-158, hep-th/9312031.

[13] Calogero F., Exactly solvable one-dimensional many-body problems, Lett. Nuovo Cimento 13 (1975), 411416.

[14] Calogero F., On a functional equation connected with integrable many-body problems, Lett. Nuovo Cimento 16 (1976), 77-80.

[15] Fehér L., Pusztai B.G., A class of Calogero type reductions of free motion on a simple Lie group, Lett. Math. Phys. 79 (2007), 263-277, math-ph/0609085.

[16] Felder G., Elliptic quantum groups, in XIth International Congress of Mathematical Physics (Paris, 1994), Int. Press, Cambridge, MA, 1995, 211-218, hep-th/9412207.

[17] Freidel L., Maillet J.M., Quadratic algebras and integrable systems, Phys. Lett. B 262 (1991), $278-284$.

[18] Gervais J.L., Neveu A., Novel triangle relation and absence of tachyons in Liouville string field theory, Nuclear Phys. B 238 (1984), 125-141.

[19] Lax P.D., Integrals of nonlinear equations of evolution and solitary waves, Comm. Pure Appl. Math. 21 (1968), 467-490.

[20] Li L.C., Parmentier S., Nonlinear Poisson structures and r-matrices, Comm. Math. Phys. 125 (1989), 545563.

[21] Magri F., Casati P., Falqui G., Pedroni M., Eight lectures on integrable systems, in Integrability of Nonlinear Systems (Pondicherry, 1996), Lecture Notes in Phys., Vol. 495, Springer, Berlin, 1997, 256-296.

[22] Maillet J.M., New integrable canonical structures in two-dimensional models, Nuclear Phys. B 269 (1986), 54-76.

[23] Moser J., Three integrable Hamiltonian systems connected with isospectral deformations, Adv. Math. 16 (1975), 197-220.

[24] Nagy Z., Avan J., Rollet G., Construction of dynamical quadratic algebras, Lett. Math. Phys. 67 (2004), 1-11, math.QA/0307026.

[25] Oevel W., Ragnisco O., R-matrices and higher Poisson brackets for integrable systems, Phys. A 161 (1989), 181-220.

[26] Olshanetsky M.A., Perelomov A.M., Classical integrable finite-dimensional systems related to Lie algebras, Phys. Rep. 71 (1981), 313-400.

[27] Pusztai B.G., On the $r$-matrix structure of the hyperbolic $B C_{n}$ model, arXiv:1205.1029.

[28] Ruijsenaars S.N.M., Schneider H., A new class of integrable systems and its relation to solitons, Ann. Physics 170 (1986), 370-405.

[29] Semenov-Tjan-Shanskii M.A., What is a classical r-matrix?, Funct. Anal. Appl. 17 (1983), 259-272.

[30] Sklyanin E.K., Some algebraic structures connected with the Yang-Baxter equation, Funct. Anal. Appl. 16 (1982), 263-270.

[31] Suris Yu.B., Why is the Ruijsenaars-Schneider hierarchy governed by the same $R$-operator as the CalogeroMoser one?, Phys. Lett. A 225 (1997), 253-262, hep-th/9602160.

[32] Xu P., Quantum dynamical Yang-Baxter equation over a nonabelian base, Comm. Math. Phys. 226 (2002), 475-495, math.QA/0104071. 